\title{
Le micro-sablage amélaire
}

\author{
Sarah Mehd*, Marie-Charlotte Mano, Olivier Sorel, Guy Cathelineau \\ Centre de soins dentaires, 2 place Pasteur, 35000 Rennes, France
}

MOTS CLÉS :

Émail /

Mordançage /

Sablage /

Ultrastructure

KEYWORDS:

Enamel/

Acid etching /

Air abrasion /

Ultrastructure

\begin{abstract}
RÉSUMÉ - La préparation de l'émail (élimination de la pellicule acquise exogène et création d'un micro-relief) est une étape indispensable avant tout collage d'attaches orthodontiques. Le produit le plus couramment utilisé en clinique orthodontique est la résine composite dont l'adhésif nécessite un mordançage à l'acide, afin d'obtenir des forces d'adhésion suffisantes. De nombreuses études ont cherché à objectiver les répercussions du mordançage sur l'émail, ainsi que les altérations conséquentes au décollage des attaches. Grâce au développement d'autres adhésifs comme les verres ionomères qui se lient chimiquement à l'émail, de nouveaux moyens de préparation amélaire sont apparus, notamment le sablage avec de la poudre d'oxyde d'alumine calibrée. Celui-ci consiste en une préparation mécanique de la dent, ce qui évite les effets néfastes des agents mordançants. En choisissant convenablement les différents paramètres de sablage (pression, temps et quantité de poudre), l'état de surface de l'émail semble moins altérée par rapport à une préparation par mordançage et l'adhésion des attaches reste supérieure aux valeurs minimales requises pour un traitement. Les résultats obtenus selon différentes études présentées indiquent donc que le sablage amélaire peut constituer une alternative crédible à la préparation par mordançage et permettre un collage efficace tout en étant plus respectueux de l'épaisseur de l'émail.
\end{abstract}

ABSTRACT - Enamel conditioning (elimination of dental plaque and creation of an irregular surface) is an essential step before bonding of orthodontic brackets. The most popular procedure in our practice is bonding with resin which requires enamel etching in order to get enough shear bond strength. Many studies have tried to evaluate the effects of enamel bonding using the acid-etching procedure as well as the changes caused by detachment of brackets. Thanks to the development of other adhesives such as glass ionomer cements which chemically bind to the enamel, new enamel conditioning methods appeared, in particular sandblasting with aluminium oxide particles. This technique is a mechanical preparation of the tooth that avoids the harmful effects of acid products. By suitably choosing the parameters of sandblasting (pressure, time and quantity of powder), enamel loss is lower than with the acid-etch procedure and the surface of the enamel seems less affected. However the bond strength remains superior to the values required for treatment. The presented results indicate that enamel sandblasting can be considered as an alternative for the acid-etching technique currently used in orthodontic practice because it creates sufficient strength and respects enamel thickness better.

\section{Introduction}

En orthodontie, comme en dentisterie restauratrice, la préparation amélaire est devenue une étape indispensable avant tout collage; elle permet une élimination de la pellicule acquise exogène et la création d'un micro-relief nécessaire à la pénétration du produit de collage. De nombreuses études ont été réalisées pour déterminer les moyens ainsi que les conditions optimales permettant d'obtenir les

*Auteur pour correspondance : sa.me@hotmail.fr 
qualités souhaitées pour un collage efficace, en préservant au mieux la dent concernée.

Le moyen de préparation amélaire le plus courant est le mordançage. Cependant, des dommages irréversibles ont été constatés sur l'émail suite à cette opération, du fait de l'attaque acide. Les laboratoires ont proposé et développé d'autres méthodes de préparation amélaire ayant pour but d'obtenir un état de surface compatible avec un collage efficace, en étant moins nuisible vis-à-vis de l'organe dentaire.

Nous proposons dans cet article d'évaluer l'une de ces techniques de préparation amélaire: le sablage. Au préalable, il est important de faire un état des lieux concernant les effets sur l'émail d'un mordançage par divers acides. Nous exposerons par la suite les résultats d'une étude analysant les différents paramètres du sablage et la conséquence de leurs variations sur l'état de surface et la profondeur de la préparation amélaire. Enfin, nous examinerons les forces obtenues après collage avec un ciment verre ionomère modifié par adjonction de résine (CVIMAR) pour différentes préparations de l'émail afin de déterminer si le sablage amélaire constitue une alternative crédible à la préparation par mordançage.

\section{Effets des préparations amélaires avec des agents déminéralisants}

Lémail dentaire est une structure très minéralisée, c'est pourquoi il est susceptible d'être attaqué par des solutions acides, comme l'acide orthophosphorique. L'hétérogénéité de structure entraîne des variations locales de solubilité. De ce fait, toute attaque déminéralisante induit des reliefs particuliers, permettant de favoriser l'ancrage de résine sur la surface traitée. Depuis quelques années, les chercheurs tentent de trouver de nouveaux moyens pour préparer l'émail et permettre un collage efficace tout en préservant au maximum le tissu dentaire. C'est ainsi qu'ont mûri des idées sur des pré-traitements utilisant d'autres solutions acides (maléique, polyacrylique... ) ou encore sur des préparations par sablage amélaire.

Quelle que soit la méthode retenue, le but est de créer des micro-rétentions allant de 5 à $10 \mu \mathrm{m}$, celles-ci permettant la pénétration du produit de collage et une rétention mécanique par imbrication entre l'émail et le matériau de collage formant la couche hybride.
De nombreux acides ont été utilisés pour mordancer l'émail, parmi lesquels l'acide chlorhydrique, l'acide citrique, l'acide formique... Cependant, le plus utilisé actuellement reste l'acide orthophosphorique.

Ce mode de préparation a été décrit pour la première fois en 1955 par Buonocore [3] en vue de sceller les sillons susceptibles à la carie. En effet, les résines composites n'établissant pas de liaisons chimiques avec l'émail, il proposa de mordancer l'émail avec de l'acide afin de créer une adhésion basée sur un ancrage mécanique. Ce conditionnement fut ensuite étendu à l'odontologie conservatrice, où les restaurations adhésives prenaient de l'essor. Par la suite, en 1964, Newman [12] étendit l'utilisation du mordançage à l'orthodontie pour coller les brackets à l'émail.

\subsection{Effets sur l'émail du mordançage à l'acide orthophosphorique}

Nous verrons quatre aspects des conséquences de l'application de cet acide

- l'ultrastructure,

- la profondeur,

- les effets sur la pulpe,

- la force d'adhésion et l'arrachement de l'émail lors de la dépose.

\subsubsection{L'ultrastructure}

Silverstone [16] décrit trois types d'architecture :

- Le type 1 est le type d'architecture le plus rencontré. Lacide atteint préférentiellement le cœur des prismes laissant la périphérie et la substance interprismatique indemnes. Les zones dissoutes ont un diamètre de $3 \mu \mathrm{m}$; l'ensemble donne un aspect décrit en nid d'abeilles (Fig. 1).

- Le type 2 où la périphérie des prismes est altérée, tandis que la substance intra-prismatique est laissée intacte. Cette attaque s'étend le long des prismes, les individualisant les uns des autres (Fig. 2)

- Le type 3 correspond à un mode de dissolution anarchique, où substance inter- et intra-prismatique sont toutes deux touchées. Il existe des irrégularités de surface de l'émail, mais la surface assez peu réactive n'adopte aucune des deux configurations précédemment décrites. 


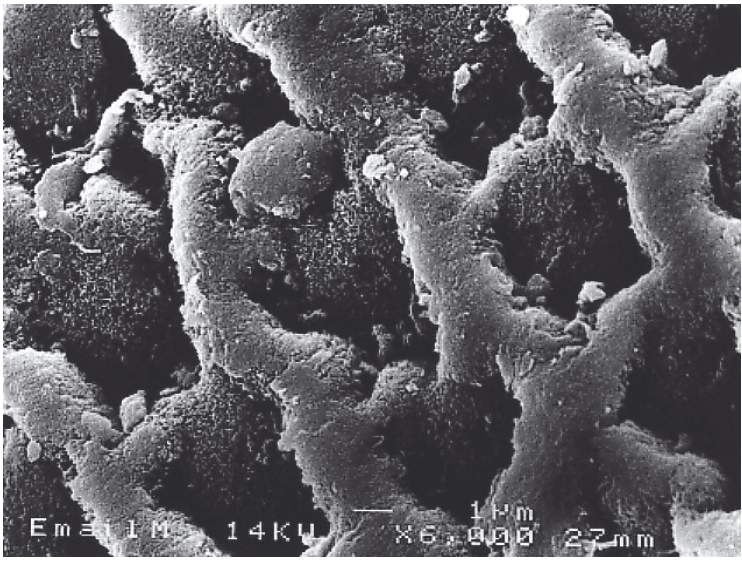

Figure 1

Type 1 de Silverstone.

\subsubsection{La profondeur}

Il existe, selon Goldberg [7], trois zones au niveau de l'émail (Fig. 3):

- une première zone d'environ $10 \mu \mathrm{m}$ en surface qui correspond à de l'émail aprismatique,

- une seconde zone sous-jacente d'environ $20 \mu \mathrm{m}$, dite poreuse, avec des modifications histologiques déterminant différents types de reliefs décrits par Silverstone [16],

- une troisième zone, également de $20 \mu \mathrm{m}$ et poreuse, mais dans laquelle il n'existe pas de modifications histologiques et dans laquelle les prolongements de résine peuvent pénétrer.

\subsubsection{Les effets sur la pulpe}

Pour Auther [1], après un traitement à l'acide et donc une déminéralisation chimique, il se produit des modifications au niveau de l'émail, mais aussi au niveau de la dentine et de la pulpe. Il existe en effet des perturbations chimiques marquées par la propagation d'une onde de phosphore qui atteint la pulpe au bout de 30 jours. Après 36 jours, l'équilibre est rétabli du côté mordancé alors qu'un déséquilibre apparaît du côté opposé.

\subsubsection{La force d'adhésion et les pertes d'émail à la dépose des attaches}

Nous pouvons définir la force d'adhésion en orthodontie comme la résistance aux contraintes liées

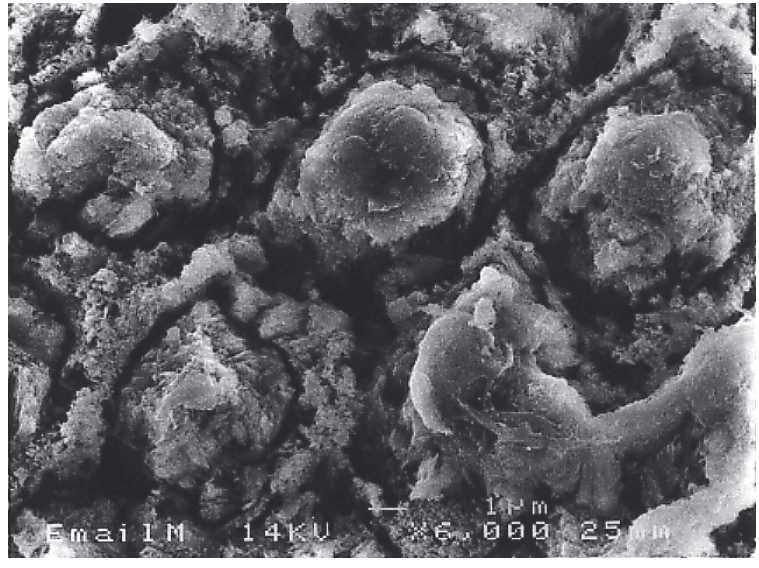

Figure 2

Type 2 de Silverstone.

aux activations pendant le traitement, à la mastication, aux parafonctions. Par ailleurs, lors de la dépose des attaches, il faut éviter toute lésion iatrogène, tout arrachement de prismes d'émail et toute fracture de substrats comme la céramique.

Osorio, et al. [14], faisant référence aux travaux de Reynolds, définissent qu'une force minimale de 6 à $8 \mathrm{MPa}$ est nécessaire et suffisante. Or, toutes les études récentes testant les forces d'adhésion des composites ou des ciments verre ionomère modifiés par adjonction de résine (CVIMAR), suite à une préparation amélaire par mordançage, montrent des niveaux de force bien supérieurs à ceux que requiert un traitement orthodontique dans des conditions habituelles. On peut, par exemple, se référer aux résultats des travaux de l'équipe de Hitmi [9], présentés dans la figure 4.

Par ailleurs, pour quantifier la perte amélaire, il est possible d'évaluer l'émail restant sur l'intrados du bracket et la quantité de colle restant sur la surface dentaire. Ainsi, deux indices ont été créés pour mesurer le taux d'émail perdu lors de la dépose des attaches:

- Les analyses qualitative et quantitative du décollement amélaire sont mesurées par l'index EDI (Enamel Detachment Index) (Fig. 5a). Lindex EDI correspond au taux d'émail présent sur l'intrados du bracket.

- Lanalyse de l'interface de rupture est mesurée par l'index ARI (Adhesive Remnant Index) (Fig. 5b). Lindex ARI est proportionnel à la quantité de résine persistant sur la dent; plus cette quantité est importante plus l'ARI est élevé. 


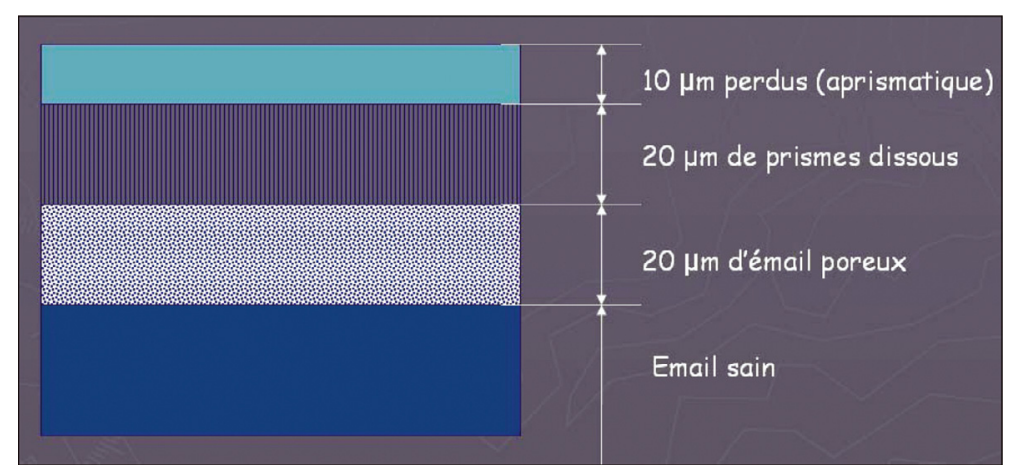

Figure 3

Structure de l'émail suite à un mordançage.

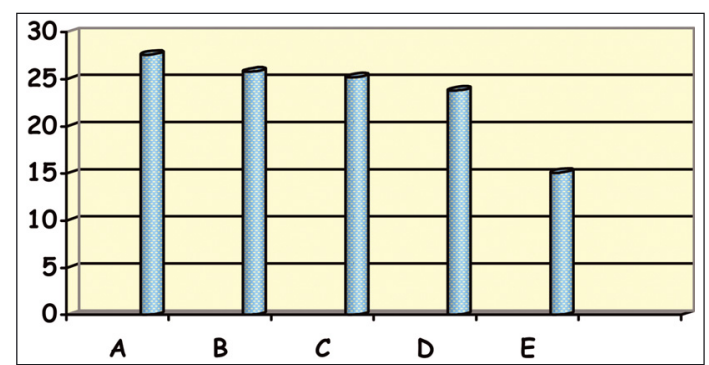

a

\begin{tabular}{|c|c|}
\hline Système de collage testé & $\begin{array}{c}\text { Moyenne +- } \\
\text { écart type en MPa }\end{array}$ \\
\hline $\begin{array}{c}\text { A :Acide phosphorique + } \\
\text { Composite hydrophobe }\end{array}$ & $27,6+-7,2$ \\
\hline $\begin{array}{c}\text { B : Self-etching + } \\
\text { Composite }\end{array}$ & $25,8+-10,1$ \\
\hline $\begin{array}{c}\text { C : Self-etching + } \\
\text { CVIMAR }\end{array}$ & $25,2+-6,9$ \\
\hline $\begin{array}{c}\text { D : Acide phosphorique + } \\
\text { CVIMAR }\end{array}$ & $23,8+-10,1$ \\
\hline $\begin{array}{c}\text { E : Acide polyacrylique + } \\
\text { CVIMAR }\end{array}$ & $15,1+-7,8$ \\
\hline
\end{tabular}

b

Figure 4

Résultats de l'étude de L. Hitmi [9] sur l'adhérence à l'émail des attaches orthodontiques collées à l'aide d'une association d'adhésif automordançant (Transbond Self etching Primer) et de CVIMAR (Fuji LC).

Dans les études de Sorel, et al. [19-22], les intrados des attaches Discovery (Fig. 6a) ont été examinés après mordançage de la surface amélaire et collage au composite. Les images obtenues montrent la présence d'émail sur l'intrados des brackets examinés (Fig. 6b). Notons que les zones de décollement sont souvent mixtes : émail/colle, rupture cohésive, colle sur l'intrados du bracket.
En résumé, le mordançage a comme effets néfastes:

- un risque carieux,

- une répercussion sur l'équilibre de l'organe dentino-pulpaire,

- une perte d'émail irréversible,

- des zones de décollement souvent mixte,

- une persistance des prolongements de résine.

Des recommandations ont ainsi été données pour respecter au mieux la surface amélaire :

- le respect du protocole de collage,

- la motivation du patient,

- les précautions nécessaires lors de la dépose de l'appareil,

- la suppression des résidus de résine à la fin du traitement.

\section{Effets sur l'émail du mordançage à d'autres acides}

\subsection{Acide maléique}

Lacide maléique est un acide organique faible. Lacide maléique [8] à $10 \%$ enlève significativement moins d'émail que l'acide orthophosphorique à $35 \%$. La force d'adhésion [10] est comparable pour un mordançage de $15 \mathrm{~s}$ avec de l'acide orthophosphorique à $35 \%$ ou de l'acide maléique à $10 \%$, à savoir alternativement $15,3 \mathrm{MPa} \pm 5,5$ et $15,8 \mathrm{Mpa} \pm 5,9$.

Même si l'acide maléique engendre une déminéralisation nettement moindre de l'émail que l'acide orthophosphorique, il altère malgré tout la qualité de l'émail en attaquant notamment la couche superficielle où le fluor a la plus grande concentration. 


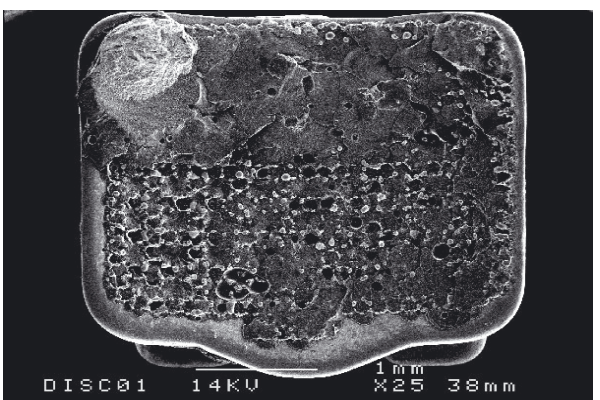

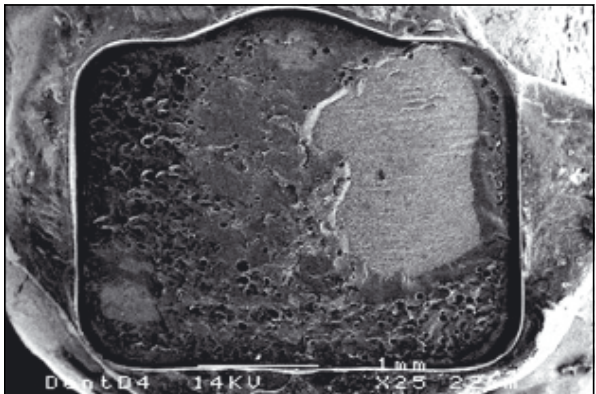

b

Figure 5

(a) Photographie d'un intrados de bracket après dépose. Dans l'angle supérieur gauche, nous notons la présence d'émail arraché à la surface dentaire lors de la dépose. La surface occupée par cet émail permet de calculer l'index EDI. (b) Photographie d'une surface amélaire après dépose d'un bracket. La surface occupée par la résine résiduelle permet de calculer l'index ARI. (D'après Sorel [22].)

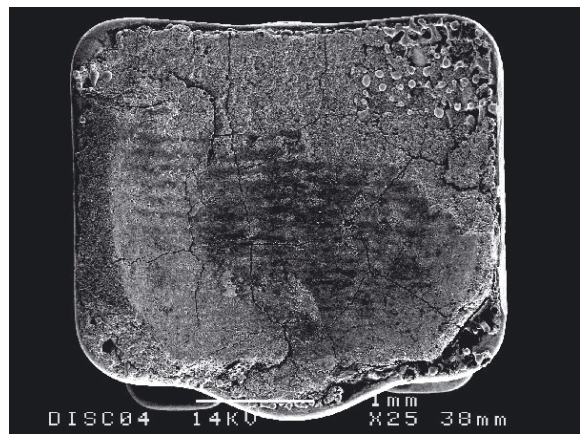

a

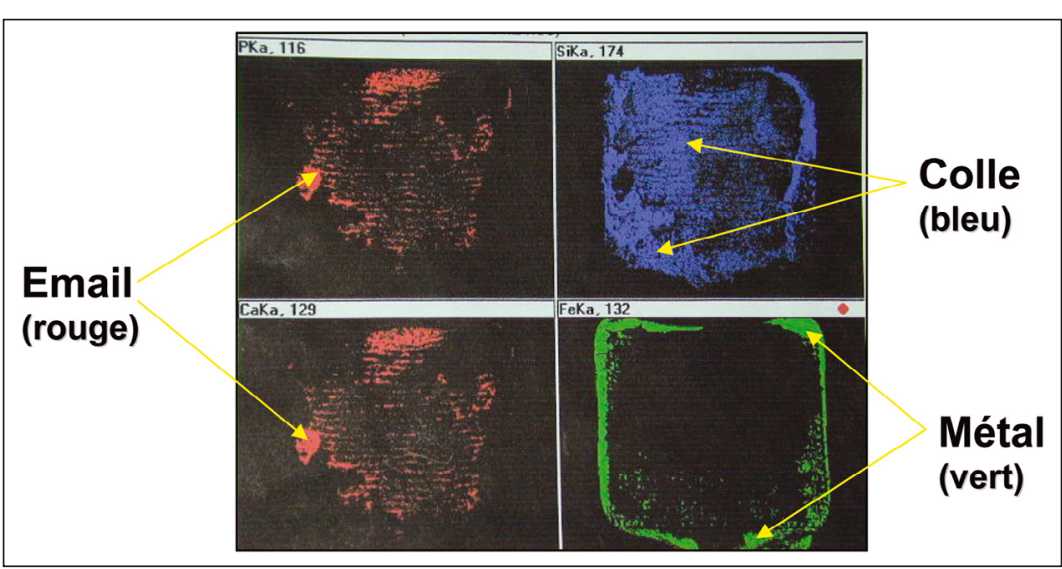

b

Figure 6

(a) Intrados d'une attache Discovery collée avec du composite, après mordançage de la surface amélaire. (b) Analyse de l'intrados de cette attache.

\subsection{Acide polyacrylique}

Ce sont Smith et Cartz, auxquels Farquhar, et al. [5] se réfèrent, qui ont eu l'idée en 1973 de créer un relief rétentif qui ne se formerait pas dans l'émail, mais à la surface de ce dernier, en se liant chimiquement à lui. Ils ont été les premiers à exposer cette méthode : ils ont pu observer, suite à l'application sur l'émail d'une solution d'acide polyacrylique contenant des ions sulfates, l'apparition de dépôts cristallins blanchâtres qui ont été ensuite identifiés comme étant du gypse. La technique dite du Crystal bonding s'appuie donc sur la création d'une surface cristalline rétentive avec des cristaux qui se lient fermement à l'émail, produisant un moyen de rétention mécanique pour la résine. Ces cristaux fortement liés à la surface amélaire semblent permettre le collage d'une résine en utilisant le même protocole que celui utilisé avec l'acide orthophosphorique. En 1979, Smith et Maijer [17] ont mis au point une solution commercialisée qui a été retirée du marché en 1986, après avoir été déclarée inapte à un emploi clinique quotidien par certaines études. Depuis, d'autres préparations commerciales ont vu le jour et sont encore sur le marché (Fig. 7a et 7b).

Les expériences réalisées par Smith et Maijer [18] en 1986 ont montré, par l'analyse chimique des ions calcium libérés après mordançage avec l'acide polyacrylique, que la perte amélaire représentait environ un sixième de celle induite par un mordançage à l'acide orthophosphorique à $50 \%$. Ceci s'explique 


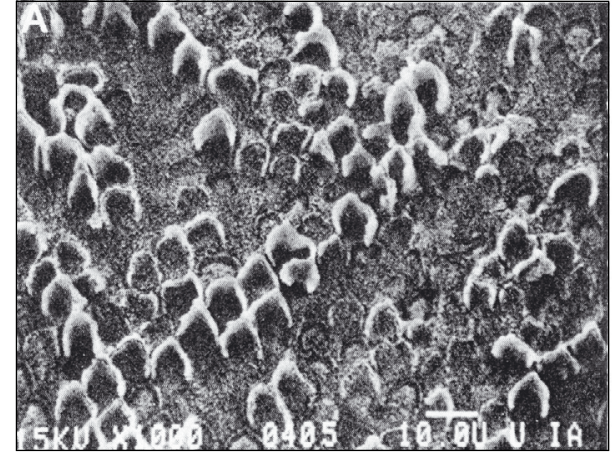

a

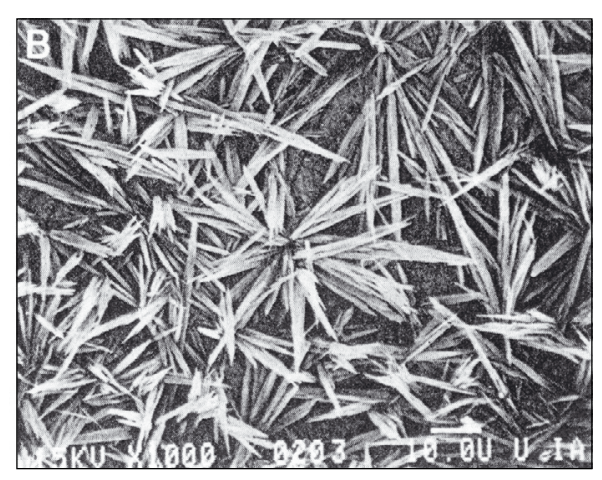

Figure 7

(a) Acide orthophosphorique à $37 \%$ pendant $30 \mathrm{~s}$ (× 1000). (b) Acide polyacrylique $(\times 1000)$. (D'après Bishara, et al. [2].)

par la formation rapide des cristaux de dihydrate de sulfate de calcium, qui réduit la pénétration de l'acide et qui limite la libération d'ions calcium depuis la surface amélaire. De plus, l'acide polyacrylique ayant un poids moléculaire beaucoup plus important que celui de l'acide phosphorique, il pénètre moins profondément dans les sites mordancés.

Pour l'acide polyacrylique, les valeurs concernant les forces d'adhésion sont faibles, elles restent néanmoins très légèrement supérieures ou équivalentes aux normes requises, ce qui permettrait une utilisation clinique tout en préservant l'émail. Ce mode de préparation peut être bénéfique au niveau des dents maxillaires antérieures lorsqu'une force de faible intensité doit être appliquée. Cependant, un bracket collé suite à une préparation de l'émail par un acide polyacrylique ne doit pas être soumis à une force excessive étant donné que la force d'adhésion qu'il procure frôlerait souvent la limite admise comme acceptable par Reynolds (cité en [14]).

Cette technique de préparation est maintenant développée essentiellement pour les collages des brackets en céramique, et notamment pour améliorer l'adhérence des ciments verre ionomère.

Lacide polyacrylique permet d'endommager le moins possible la couche superficielle d'émail riche en ions fluorures, d'engendrer peu, voire même pas, de prolongements de résine persistant dans l'émail, d'obtenir une force d'adhésion suffisante pour une utilisation clinique à condition que les forces ne soient pas excessives, une dépose des brackets aisée et un nettoyage de la dent facile et rapide et, enfin, la possibilité d'incorporer dans l'avenir des ions fluorures ou des agents anti-plaque afin d'avoir une action anti-cariogène.

Toutefois, il s'agit toujours d'une préparation chimique et les inconvénients tels que la diffusion de l'acide persistent. Aussi, une préparation exclusivement mécanique permettrait de supprimer les défauts liés à la présence d'acide, c'est cette possibilité qu'offre le sablage.

\section{Préparation de l'émail par micro-sablage}

\subsection{Historique}

Le concept du sablage est relativement ancien puisqu'il a été décrit pour la première fois en 1943 par Robert Black (cité en [6]). Ce dernier préconisait son utilisation pour la préparation des cavités en odontologie conservatrice. En effet, son usage était beaucoup plus confortable tant pour le patient que pour le praticien, comparé aux instruments rotatifs à basses vitesses, employés alors. Il a donc remporté un large succès au cours de cette décennie. Par la suite, cette technique a été détrônée avec l'avènement des turbines.

En 1993, Zachrisson [24] propose d'utiliser le sablage pour coller sur l'or, l'amalgame et la porcelaine.

\subsection{Principes du sablage et paramètres de travail}

\subsubsection{Principes}

Le sablage est une préparation mécanique de l'émail, contrairement au mordançage qui est une préparation chimique (dissolution chimique des cristaux d'hydroxyapatite de l'émail). 


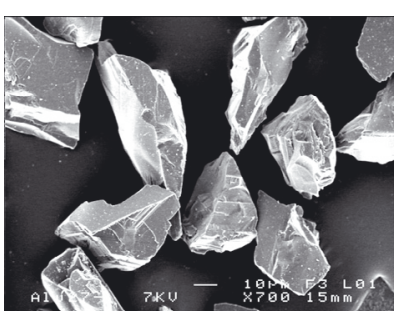

a

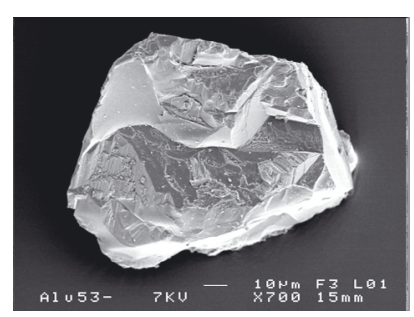

b

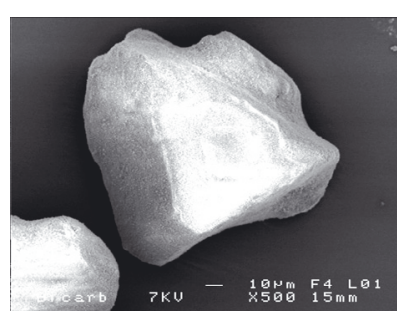

C

Figure 8

Particules projetées. (a) Oxyde d'alumine, $29 \mu \mathrm{m}$. (b) Oxyde d'alumine, $50 \mu \mathrm{m}$. (c) Bicarbonate de sodium.

Le sablage consiste à projeter des particules à haute vitesse dans un flux d'air sec ou dans un brouillard d'eau.

Cette technique est basée sur la théorie de l'énergie cinétique Ec :

$$
E c=\frac{1}{2} m V^{2}
$$

- $m$ : masse des particules tel que $m=\delta$, avec $\delta$ : densité de la particule, $v$ : volume de la particule tel que $v=4 / 3 \pi \mathrm{r}^{3}$,

$-V$ : vitesse d'expulsion (liée à la pression).

Plus une substance est dure, plus la vitesse de coupe est rapide.

À l'inverse, plus la substance est molle, moins la coupe se fait vite.

\subsubsection{Paramètres de travail}

- Les particules projetées

Elles peuvent être de différentes natures et de différentes tailles :

- billes de verre au laboratoire,

- oxyde d'alumine (trois tailles commercialisées : $29 \mu \mathrm{m}, 50 \mu \mathrm{m}, 90 \mu \mathrm{m}$ ) (Fig. 8a et 8b),

- bicarbonate de sodium (Fig. 8c).

- La pression

La pression est généralement réglée manuellement. L'échelle de graduation est le bar.

- La quantité de poudre

Celle-ci est également réglée manuellement pour la plupart des appareils.

- La buse (Fig. 9)

Différents types de buses existent, variant par leur diamètre d'éjection. Plus celui-ci est petit, plus la vitesse d'éjection augmente. Plus le jet est concentré,

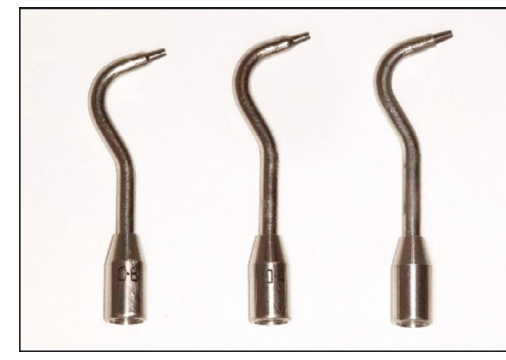

Figure 9

Buses de 0,8 , puis 0,6 et $0,4 \mathrm{~mm}$.

plus la coupe est puissante et précise. Nous utilisons en clinique la buse de 0,8 $\mathrm{mm}$ la plus ouverte, pour avoir une action de surface et non ponctuelle.

- Le temps d'application

Ce temps a une importance primordiale sur le sablage. L'étude au profilomètre, que l'on développera par la suite, montre que les reliefs obtenus n'augmentent pas avec le temps d'application. En revanche, plus le temps augmente, plus on creuse l'émail. Nous recommandons en clinique 2 à $3 \mathrm{~s}$ par dent.

\subsection{Ultrastructure et profondeur obtenues après sablage}

Pour étudier l'ultrastructure de la surface amélaire, ainsi que la profondeur du micro-relief créé, nous allons exposer les résultats d'une étude réalisée in vitro dans le cadre d'une thèse doctorale [11] analysant les effets d'un sablage avec des particules d'oxyde d'alumine de $29 \mu \mathrm{m}$.

\subsubsection{Matériels et méthodes}

Dix-huit prémolaires fraîchement extraites, dépourvues de toute restauration et de toute affection carieuse, ont été conservées dans de l'eau. La face 


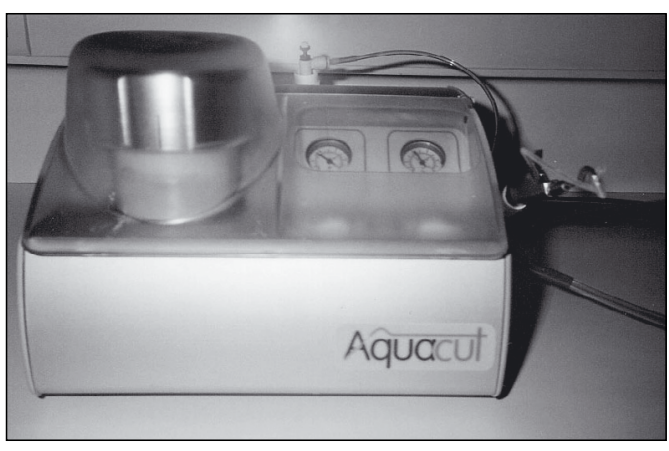

Figure 10

Aquacut $^{\circledR}$ : appareil employé pour le sablage.

vestibulaire de chacune de ces dents a été sectionnée avec un disque en carborundum de façon à ce que la partie linguale de chacun de ces échantillons soit parallèle à la face vestibulaire. Ces échantillons ont ensuite été divisés en deux groupes:

- Les surfaces des dents du premier groupe ont été aplanies à l'aide d'un disque abrasif, puis polies avec une pointe en caoutchouc.

- Les dents du second groupe n'ont, en revanche, subi aucun prétraitement.

La surface de chacun de ces échantillons fut alors recouverte d'une feuille de plomb évidée en son centre d'un carré de $14 \mathrm{~mm}^{2}$ correspondant à la surface d'un bracket, puis les surfaces des deux groupes ont subi un sablage par une poudre d'oxyde d'alumine constituée de grains de $28 \mu \mathrm{m}$ (Fig. 10). La tête de l'instrument étant maintenue perpendiculairement à $1 \mathrm{~mm}$ de la surface et manipulée avec un mouvement de va-et-vient transversal par rapport à la surface vestibulaire, afin de balayer l'ensemble de la surface.

Les sablages différaient dans les trois paramètres étudiés, à savoir ceux de temps, de pression et de quantité de poudre utilisée. Le tableau 1 résume les différents traitements subis par les échantillons.

Les échantillons seront par la suite désignés par la valeur des trois variables temps, pression et poudre. Ainsi, l'échantillon 1 sera nommé «2-4-4». Les échantillons furent ensuite rincés afin d'éliminer les grains d'alumine.

Les faces issues du groupe 1 ont été passées au profilomètre Mitutoyo ${ }^{\circledR}$, après avoir été fixées sur une surface plane avec de la cire Lactona. Après analyse des reliefs obtenus, ces faces ont été passées au microscope électronique à balayage (MEB), afin de
Tableau 1

Variables «temps, pression et quantité de poudre » en fonction des échantillons.

\begin{tabular}{|c|c|c|c|}
\hline Échantillon & Temps (s) & Pression (bars) & Poudre \\
\hline 1 & 2 & 4 & 4 \\
\hline 2 & 4 & 2 & 2 \\
\hline 3 & 4 & 2 & 4 \\
\hline 4 & 4 & 4 & 4 \\
\hline 5 & 6 & 2 & 2 \\
\hline 6 & 6 & 2 & 4 \\
\hline 7 & 8 & 2 & 2 \\
\hline 8 & 8 & 2 & 4 \\
\hline 9 & 10 & 2 & 2 \\
\hline
\end{tabular}

s'assurer de la concordance entre les images obtenues à partir du premier groupe et celles obtenues à partir du deuxième groupe.

Cette étape avait pour objectifs, d'une part, de déterminer la profondeur des reliefs créés par les différents traitements et, d'autre part, d'en étudier le profil et donc de mesurer :

- l'écart moyen arithmétique de profil (de rugosité) Ra, représentant la moyenne arithmétique des valeurs absolues des écarts de chaque pic par rapport à la ligne moyenne;

- la hauteur maximum du profil Ry représentant, en $\mu \mathrm{m}$, la distance entre le pic le plus haut et le pic le plus bas du tracé (Fig. 1la);

- la hauteur des irrégularités sur dix points Rz représentant, en $\mu \mathrm{m}$, la moyenne de hauteur des cinq points les plus hauts et des cinq points les plus bas du tracé (Fig. 11b).

Les échantillons issus du groupe 2 furent passés au microscope électronique à balayage, afin d'observer l'état de surface créé par chacun des traitements.

\subsubsection{Résultats}

- Résultats obtenus au profilomètre (Tab. 2)

Ces résultats montrent que l'état de surface dépend effectivement des trois paramètres que l'on a fait varier à savoir le temps, la pression et la quantité de poudre et ceci, à des degrés propres à chacun (Fig. 12a à 12c).

Nous observons que les valeurs Rz (hauteur des irrégularités sur dix points) et Ra (écart moyen arithmétique de profil) augmentent avec le facteur temps jusqu'à $6 \mathrm{~s}$ d'exposition, puis la courbe s'inverse, leurs valeurs décroissant avec le temps. Cette étude 


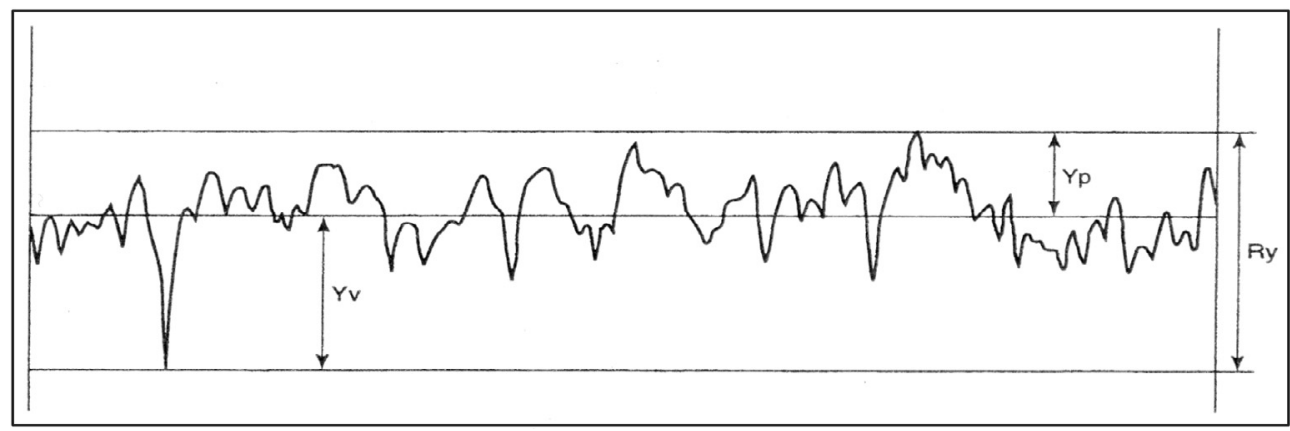

a

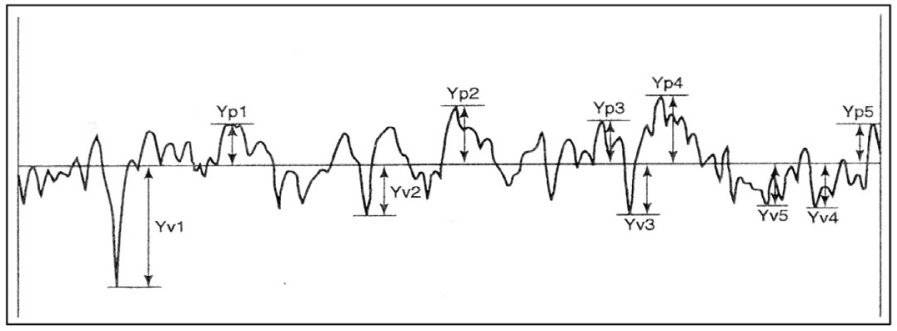

b

Figure 11

(a) Mesure de la hauteur maximum du profil Ry. (b) Mesure de la hauteur des irrégularités sur dix points Rz.

Tableau 2

Résultats obtenus au profilomètre.

\begin{tabular}{|c|c|c|c|c|}
\hline Échantillon & Sens de passage & $\mathrm{Ra}(\mu \mathrm{m})$ & $\mathrm{Ry}(\mu \mathrm{m})$ & $\mathrm{Rz}(\mu \mathrm{m})$ \\
\hline \multirow{2}{*}{-4-4 } & Vertical & 2,72 & 15,20 & 9,48 \\
\cline { 2 - 5 } & Horizontal & 1,46 & 14,35 & 9,34 \\
\hline \multirow{2}{*}{$4-2-2$} & Vertical & 0,86 & 7,81 & 4,27 \\
\cline { 2 - 5 } & Horizontal & 0,73 & 6,69 & 4,55 \\
\hline \multirow{2}{*}{$4-2-4$} & Vertical & 0,86 & 7,94 & 4,18 \\
\cline { 2 - 5 } & Horizontal & 0,60 & 8,11 & 4,69 \\
\hline \multirow{2}{*}{$4-4-4$} & Vertical & 1,74 & 23,08 & 10,47 \\
\cline { 2 - 5 } & Horizontal & 0,92 & 6,83 & 5,79 \\
\hline \multirow{2}{*}{$6-2-2$} & Vertical & 0,99 & 7,82 & 5,58 \\
\cline { 2 - 5 } & Horizontal & 0,83 & 6,04 & 4,34 \\
\hline \multirow{2}{*}{$6-2-4$} & Vertical & 1,22 & 10,51 & 6,24 \\
\cline { 2 - 5 } & Horizontal & 1,35 & 11,52 & 6,83 \\
\hline \multirow{2}{*}{$8-2-2$} & Vertical & 0,53 & 3,83 & 2,94 \\
\cline { 2 - 5 } & Horizontal & 0,65 & 4,27 & 3,02 \\
\hline \multirow{2}{*}{$8-2-4$} & Vertical & 4,32 & 21,58 & 11,71 \\
\cline { 2 - 5 } & Horizontal & 0,89 & 5,24 & 4,69 \\
\hline \multirow{2}{*}{ 10-2-2 } & Vertical & 0,45 & 7,18 & 3,84 \\
\cline { 2 - 5 } & Horizontal & 0,53 & 4,92 & 3,17 \\
\hline
\end{tabular}




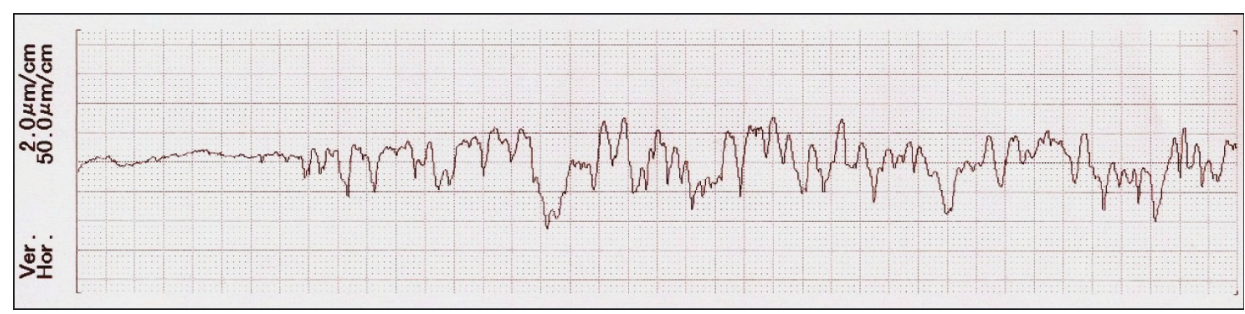

a

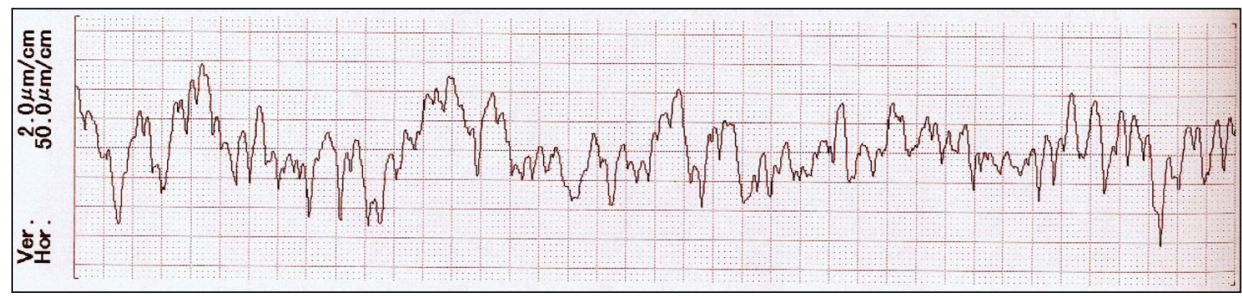

b

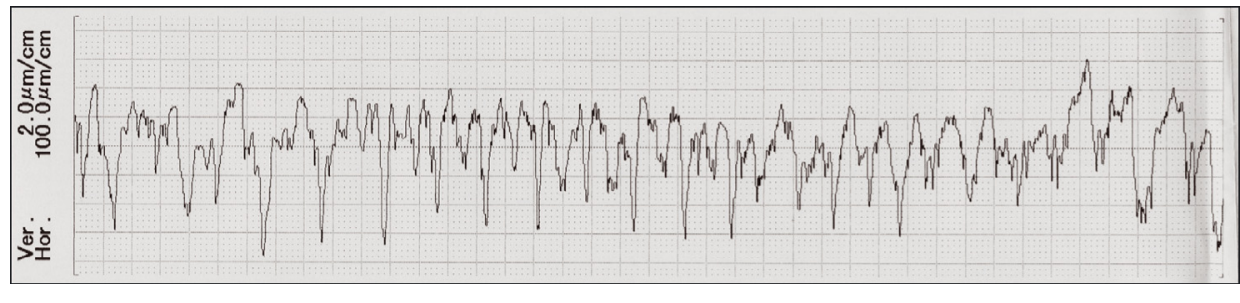

C

Figure 12

(a) Graphique obtenu pour l'échantillon 4-2-2. La limite de la surface sablée est nette. (b) Graphique obtenu pour l'échantillon 6-2-2. Les pics ont une plus grande amplitude. (c) Graphique obtenu pour l'échantillon 4-4-4. Les pics sont beaucoup plus rapprochés que dans les précédents graphiques.

montre également qu'une augmentation de la pression entraîne un accroissement considérable de la profondeur des reliefs puisque les valeurs les plus fortes sont observées pour une pression de 4 bars et ce, quel que soit le temps de sablage. Enfin, la quantité de poudre employée ne semble pas avoir beaucoup d'influence sur la profondeur du relief. Il semble cependant que la quantité de poudre joue un rôle dans la fréquence des pics.

Ceci tend à montrer que l'on obtiendrait un relief optimal pour une valeur précise de temps et de pression.

\section{- Résultats obtenus avec le MEB}

Les différents échantillons du groupe 2 ont été passés au microscope électronique à balayage (Fig. 13a à 13d). Les clichés pris au MEB montrent que, pour les échantillons traités avec une pression faible et un temps court, les impacts sont moins nombreux que pour les échantillons traités avec un temps plus long ou une pression plus élevée. Les zones d'émail non atteintes ou faiblement atteintes sont donc de moins en moins visibles lorsque les variables temps et pression augmentent. À partir de $6 \mathrm{~s}$ d'ailleurs, quelle que soit la pression, les reliefs observés differrent peu les uns des autres et il devient très difficile de reconnaître les échantillons.

D'autres clichés ont été pris selon un angle de $180^{\circ}$ par rapport aux échantillons (Fig. 14). Ils ont donc permis d'avoir une idée de l'état de surface, mais n'ont pu renseigner sur la profondeur des reliefs créés. Il faut noter que, dans tous les cas, l'architecture de l'émail est complètement dénaturée, il ne reste plus aucun relief de l'état initial de l'émail.

\subsubsection{Discussion}

La présente étude a pour objectif de déterminer l'ultrastructure de l'émail sablé. Aussi, l'étude au profilomètre a-t-elle permis de déterminer la profondeur des reliefs obtenus. Toutefois, dans cette étude, il a fallu s'éloigner un peu des conditions cliniques et 


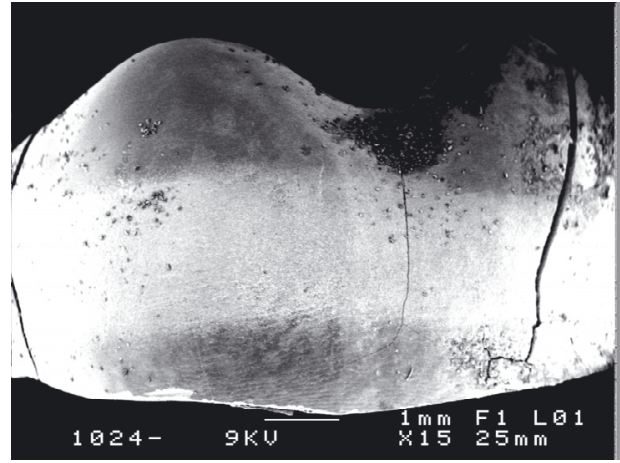

a

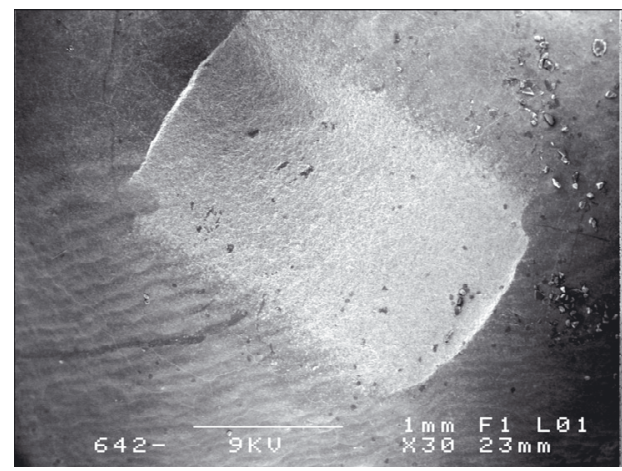

b

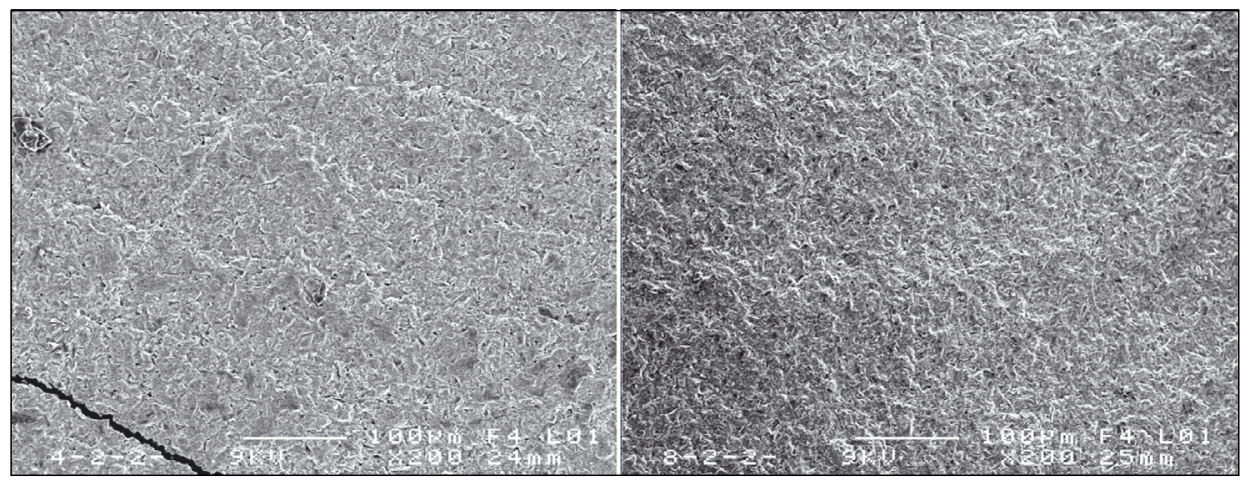

C

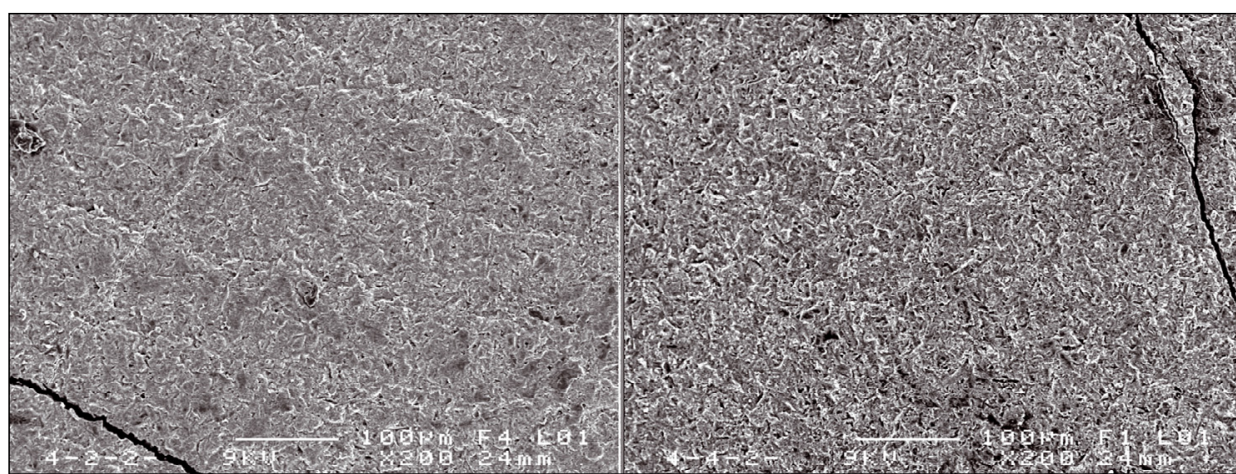

d

Figure 13

(a) Face vestibulaire d'une molaire sablée $(\times 5)$. (b) Limites de la zone de sablage sur l'échantillon 6-4-2 $(\times 30)$. Sur cette image, on note la disparition des reliefs à la superficie de l'émail au niveau de la zone sablée. (c) Comparaison entre la surface sablée pendant 4 s et celle sablée pendant $8 \mathrm{~s}(\times 200)$. (d) Comparaison entre la surface sablée avec une pression « 2 " et celle sablée avec une pression « $4 »(\times 200)$.

aplanir la surface des dents à analyser au profilomètre, leur courbure initiale empêchant toute mesure. Par conséquent, les résultats obtenus avec le profilomètre diffèrent probablement un peu de ceux trouvés en pratique courante.

D'après plusieurs analyses, aucun traitement n'engendrant plus de $100 \mu \mathrm{m}$ de perte d'émail n'est considéré comme dommageable pour l'émail [13].
Or, les valeurs obtenues dans cette étude sont toutes bien inférieures à cette limite et, par conséquent, rejoignent les conclusions de Reisner, et al. [15], ainsi que celles plus récentes de Canay [4], à savoir que le sablage ne produit pas d'altérations trop importantes pour l'émail. Toutefois, cette étude montre très clairement que la variation des paramètres temps et pression intervient considérablement sur la profondeur 


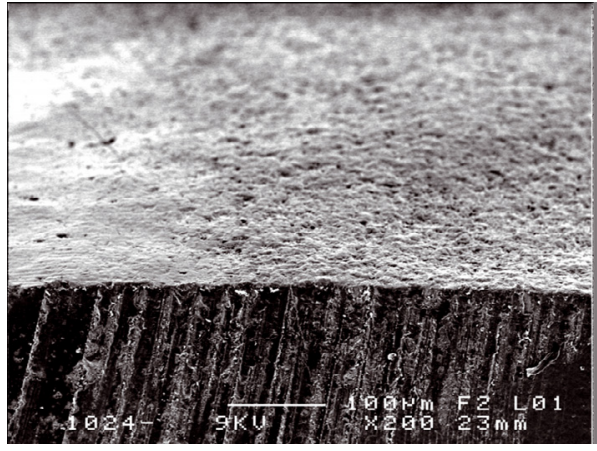

Figure 14

Échantillon 10-2-4 : image de profil (× 1000).

des reliefs réalisés. Ainsi, la profondeur créée par le sablage peut être contrôlée par le manipulateur de l'appareil en ajustant les différentes variables.

D'autre part, cette étude montre qu'augmenter la quantité de poudre projetée n'a que peu d'influence sur le relief et la profondeur; en revanche, le temps d'exposition est un paramètre important puisque jusqu'à une exposition de $6 \mathrm{~s}$ pour une pression de 2 bars, la profondeur des reliefs augmente avec le temps, puis elle décroît. Il semble donc qu'en augmentant le temps, la surface préparée s'aplanisse, devienne moins propice au collage et qu'une nouvelle couche d'émail ne commence, alors, à être altérée. La pression est également un paramètre très important à considérer, puisque c'est elle qui entraîne le plus de variations dans les profondeurs obtenues. C'est ce qu'avaient déjà fait remarquer Van Waveren Hogervorst, et al. [23].

Lobservation des images obtenues avec le MEB indique clairement que le sablage entraîne une perte de matériel organique et inorganique de la matrice amélaire et, par conséquent, une reminéralisation de cette couche d'émail sablée ne pourra se reproduire: la perte d'émail est donc définitive. Cependant, cette perte de substances organiques et inorganiques présenterait l'avantage de ne pas entraîner la persistance de prolongements de résine après le décollement des brackets.

En résumé, en allongeant la durée de sablage, la surface s'aplanit et devient moins propice au collage. Laugmentation du temps de sablage ne fait qu'ôter des couches successives d'émail et n'affecte pratiquement pas la profondeur des aspérités.

En revanche, la pression et la quantité de poudre ont une grande influence.
Nous conseillons donc de

- minimiser le temps de sablage,

- avoir une pression suffisamment forte,

- projeter une quantité de poudre importante.

Notre recherche permet d'estimer les paramètres idéaux du sablage pour l'Aquacut ${ }^{\circledR}$, comme suit :

- temps: 2 à $6 \mathrm{~s}$ d'application sur une dent non préparée,

- pression : 3 à 4 bars,

- poudre : échelle 3 à 4 .

\subsubsection{Conclusion}

D'après l'étude de données fournies par deux méthodes d'analyse de l'ultrastructure produite par le sablage amélaire, il ressort que la profondeur obtenue après un tel traitement de l'émail peut être modulée par l'utilisateur, en faisant varier pression et temps d'exposition. Ainsi employé dans de bonnes conditions, le sablage de la surface amélaire n'entraîne pas de dommages conséquents pour l'émail. De plus amples études devraient être faites pour chaque appareil commercialisé, afin de déterminer la combinaison des différents paramètres permettant d'obtenir le meilleur rapport bénéfice/risque, c'est-àdire obtenir des forces d'adhésion suffisantes, tout en préservant le plus possible l'émail.

Selon la plupart des études menées jusqu'ici, les forces d'adhésion obtenues avec des résines composites sont très largement inférieures à celles que requiert un traitement orthodontique. Cependant, avec le développement des ciments verre ionomère modifiés par adjonction de résine, les données ont changé et, comme nous allons le voir, un sablage suffit pour nettoyer la surface et créer un relief suffisamment rétentif.

\subsection{Sablage et force d'adhésion}

Comme nous l'avons déjà précisé plus haut, selon Osorio, et al. [14], une force minimale de 6 à $8 \mathrm{MPa}$ est nécessaire et suffisante dans le cadre d'un traitement orthodontique. Le sablage ne peut être considéré comme une technique fiable de préparation au collage avec de la résine (Tab. 3). Par ailleurs, le sablage avant mordançage augmente la fiabilité du collage avec de la résine; cependant, nous retrouvons tous les inconvénients liés à l'utilisation d'un acide (Tab. 4). 
Tableau 3

Résultats de l'étude d'Olsen, et al. [13].

\begin{tabular}{|c|c|c|c|}
\hline & Acide orthophosphorique & $\begin{array}{l}\text { Sablage } \\
50 \mu \mathrm{m}\end{array}$ & $\begin{array}{l}\text { Sablage } \\
90 \mu \mathrm{m}\end{array}$ \\
\hline Force & $10,4 \mathrm{MPa} \pm 2,8$ & $2,3 \mathrm{MPa} \pm 1,0$ & $3,6 \mathrm{MPa} \pm 2,2$ \\
\hline
\end{tabular}

Tableau 4

Résultats des études de Reisner [15] sur différentes préparations amélaires et de Canay [4] sur les effets du sablage sur les forces d'adhésion.

\begin{tabular}{|c|c|c|c|}
\hline & $\begin{array}{c}\text { Acide orthophosphorique } \\
\mathbf{3 0 \% - 3 0 "}\end{array}$ & $\begin{array}{c}\text { Sablage } \\
\mathbf{2 - 3 " 7 0 p s i ~} \mathbf{3 0} \boldsymbol{\mu m}\end{array}$ & $\begin{array}{c}\text { Sablage } \\
+ \\
\text { mordançage }\end{array}$ \\
\hline Force (étude de Reisner [15]) & $80,24 \mathrm{~N} \pm 2,8$ & $38,8 \mathrm{~N} \pm 16,5$ & $84,6 \mathrm{~N} \pm 23,0$ \\
\hline Force (étude de Canay [4]) & $62,7 \mathrm{~N} \pm 11,4$ & $38,0 \mathrm{~N} \pm 9,9$ & $89,3 \mathrm{~N} \pm 13,3$ \\
\hline
\end{tabular}

La force d'adhésion n'est pas suffisante lorsque la préparation amélaire se fait uniquement par microsablage, car la résine composite a besoin d'un relief plus anfractueux que celui créé par sablage. Cependant, si le couple résine/sablage n'est pas utilisable, doit-on rejeter le sablage? Non, si nous changeons l'adhésif. Les ciments verre ionomère modifié par adjonction de résine (CVIMAR) présentent l'avantage de permettre l'adhésion chimique à la structure dentaire de par leur composition. Lassociation de cette adhésion chimique et de la préparation mécanique par sablage pourrait donc constituer une alternative aux collages classiques.

Létude réalisée par Sorel [22] a comparé les forces d'adhésion obtenues avec trois types de préparation amélaire, les protocoles de collage étant identiques, à savoir un collage avec un CVIMAR (Fuji Ortho LC ${ }^{\circledR}$ ) d'attaches Discovery ${ }^{\circledR}$. Les résultats des tests de traction in vivo, classés par ordre décroissant des forces obtenues, sont les suivants :

Groupe 1 : Protocole classique (mordançage à l'acide orthophophorique 37\%) : 10,99 $\mathrm{MPa} \pm 2,26$.

Groupe 4 : Protocole par micro-sablage (variables : temps 6 - pression 3 - poudre 3) : 10,76 $\mathrm{MPa} \pm 2,04$.

Groupe 3 : Protocole par micro-sablage (variables : temps 3 - pression 3 - poudre 3) : 9,91 $\mathrm{Mpa} \pm 2,45$.

Groupe 5 : Protocole par micro-sablage (variables: temps 4 - pression 4 - poudre 4) : 9,09 Mpa $\pm 1,97$.
Groupe 2: Protocole sans mordançage (simple nettoyage avec une brossette) : 8,47 MPa $\pm 1,57$.

Le joint de colle est donc bien influencé par le type de préparation de l'émail. Les résultats des tests statistiques montrent que tous les groupes sont statistiquement différents entre eux. Le mode de préparation de la surface d'émail à coller est donc important du point de vue de l'adhésion. La moyenne d'adhésion de chaque groupe avoisine les $10 \mathrm{MPa}$ considérés comme la valeur idéale de collage. Seule la valeur moyenne d'adhésion du groupe 2 (protocole sans mordançage avec élimination de la pellicule acquise exogène avec une brossette) est légèrement inférieure.

Tous les protocoles semblent, d'après cette étude in vitro, être utilisables in vivo. En effet, tous les protocoles testés génèrent des forces de ruptures au décollement importantes. Ces forces nous semblent compatibles avec une utilisation clinique. C'est l'adhésion chimique des CVIMAR (Fuji Ortho LC) avec l'émail qui permet ces résultats; l'excellent ancrage mécanique de l'attache en est la contre-partie.

Le mordançage ne nous semble pas apporter une adhérence suffisamment supérieure compte tenu de l'effet délétère qu'il crée sur l'émail (perte d'environ $30 \mu \mathrm{m}$ au moins et systématique).

\section{Conclusion générale}

Avec l'introduction des techniques de collage pour les brackets orthodontiques, différentes approches ont été proposées pour conditionner la 
surface amélaire. La technique usuelle unanimement reconnue consiste en une application d'acide orthophosphorique préparant chimiquement et mécaniquement l'émail avant l'application d'une résine composite. Cette technique a été longuement étudiée afin de déterminer, en fonction du temps d'application et de la concentration, la combinaison idéale pour aboutir à un collage optimal avec une perte minimale d'émail après différents traitements.

D'autres approches ont été suggérées, notamment l'utilisation d'un sablage amélaire qui, au lieu de mordancer la surface amélaire, crée des macroporosités grâce à un retrait différentiel d'émail. Le sablage assure une adhérence intéressante à condition d'utiliser un CVIMAR. Les études présentées montrent qu'en déterminant les valeurs idéales de temps, pression et quantité de poudre, il était possible de produire un état de surface compatible avec un collage efficace dans le cadre d'un traitement orthodontique. Les meilleurs résultats ont été obtenus en utilisant des CVIMAR, puisque leur adhésion chimique naturelle combinée à la préparation mécanique par sablage fournit des forces d'adhésion suffisantes, tout en respectant l'émail.

Le sablage couplé au CVIMAR a des répercussions sur l'émail bien inférieures (perte d'environ $10 \mu \mathrm{m}$, moins de prolongements de résine dans l'émail) comparé au mordançage ; de plus, en un seul geste, la pellicule acquise exogène est éliminée et la surface est prête à recevoir l'adhésif. À raison de $4 \mathrm{~s}$ par dent, cela fait pour 10 dents un temps de $40 \mathrm{~s}$. Un rinçage rapide complète la préparation avant collage. En moins d'une minute, les dents sont prêtes à être collées.

\section{Bibliographie}

[1] Auther A. Aspect biologique du mordançage de l'émail, étude au MEB. Info Dential 1979:34:2965-2970.

[2] Bishara SE, Fehr DE, Jakobsen JR. A comparative study of the debonding strengths of different ceramic brackets, enamel conditioners and adhesives. Am J Orthod Dentofacial Orthop 1993;104:170-179

[3] Buonocore MG. A simple method of increasing the adhesion of acrylic filling materials to enamel surfaces. J Dent Res 1955;34:849-853.

[4] Canay S, Kocadereli I, Akca E. The effect of enamel air abrasion on the retention of bonded matallic orthodontic brackets. Am J Orthod Dentofacial Orthop 2000;117:15-19.

[5] Farquhar RB. Direct bonding comparing polyarylic acid and a phosphoric acid technic. Am J Orthod Dentofacial Orthop 1986;90:187-194
[6] Goldstein RE, Parkins FM. Air-abrasive technology: its a new role in restorative dentistery. J Am Dent Assoc 1994,125:551-557.

[7] Goldberg M. Structure de l'émail et de la dentine, effets d'agents déminéralisants et incidence sur le collage des matériaux. Actual Odontostomatol 1984;147:411-422.

[8] Hermsen RJ, Vrijhoef MMA. Loss of enamel due to etching with phosphoric or maleic acid. Dent Mat 1993;9:332-336.

[9] Hitmi L. Étude et optimisation de l'adhésion à l'émail et à la dentine. Du laboratoire à la clinique. Thèse. 2004.

[10] Holtan JR, Nystrom GP, Phelps RA, Anderson TB, Becker WS. Influence of different etchants and etching times on shear bond strength. Oper Dent 1995;20:94-99.

[11] Mehdi S, Lurquin C. Microsablage amélaire : étude expérimentale. Thèse de doctorat en odontologie. Rennes : Université de Rennes 1, 2002.

[12] Newman GV. Epoxy adhesives for orthodontic attachments: Progress Report. Am J Orthod 1965;12:90-91.

[13] Olsen ME, et al. Comparison of shear bond strength and surface structure between conventional acid etching and air-abrasion of human enamel. Am J Orthod Dentofacial Orthop 1997,112:502-506.

[14] Osorio R, Toledano M, Garcia-Godoy F. Bracket bonding with 15- or 60-second etching and adhesive remaining on enamel after debonding. Angle Orthod 1999;69:45-48.

[15] Reisner KR, et al. Enamel preparation for orthodontic bonding: a comparison between the use of sandblaster and current techniques. Am J Orthod Dentofacial Orthop 1997;111:366-373.

[16] Silverstone LM. Variation in the pattern of acid etching of human dental enamel examinated by SEM. Caries Res 1975;9:373-387.

[17] Smith DC, Maijer R. Crystal growth on the outer enamel surface: an alternative to acid etching. Am J Orthod 1986;89:183-193.

[18] Smith DC, Maijer R. A New surface treatment for bonding. Biomed Mater Res 1979;13:975-985.

[19] Sorel O, El Alam R, Chagneau F, Cathelineau G. Changes in the enamel after in vitro debonding of brackets bonded with a modified glass ionomer cement. Orthod Fr 2000;71:155-163.

[20] Sorel O, Cathelineau G. Influence of the bonding interface on bracket adhesion. Orthod Fr 2001;72:305-312.

[21] Sorel O, El Alam R, Chagneau F, Cathelineau G. Comparison of bond strength between simple foil mesh and laser sculpted base retention brackets. Am J Orthod Dentofacial Orthop 2002;122:260-266.

[22] Sorel O. La rétention structurale par laser des attaches orthodontiques : étude expérimentale. Thèse de doctorat d'université. Rennes : Université de Rennes 1, 2007.

[23] Van Waveren Hogervorst WL, Feilzer AJ, Prahl-Andersen B. The Air-abrasion technique versus the conventionnal acid-etching technique : a quantification of surface enamel loss and a comparison of shear bond strength. Am J Orthod Dentofacial Orthop 2000;117:20-26.

[24] Zachrisson BU, Buyukyilmaz T. Recent advances in bonding to gold, amalgam and porcelain. J Clin Orthod 1993;27:661-674. 